\title{
Comparação das comunidades de Sminthuroidea e Onychiuridae (Collembola) entre plantio direto em três níveis de fertilidade, plantio convencional e um ecossistema natural (campo nativo) em Ponta Grossa, Paraná, Brasil
}

\author{
Klaus Dieter Sautter ${ }^{1}$ \\ Honório Roberto dos Santos ${ }^{2}$ \\ Paulo Justiniano Ribeiro Júnior ${ }^{3}$
}

\begin{abstract}
Comparison of the communities of Sminthuroidea and Onychiuridae (Collembola) among no-tillage in three levels of fertility, conventional tillage and a natural ecosystem (native grassland) in Ponta Grossa, Paraná, Brazil. This work had as objective to compare the communities of Sminthuroidea and Onychiuridae (Collembola) among no-tillage in three fertility levels, conventional tillage and a natural ecosystem (native grassland). In the conventional tillage and in the no-tillage in area of medium fertility, the community of Sminthuroidea had regular fluctuation along the period of the experiment, but with low densities. The no-tillage in area of high fertility presented a populational pick in the autumn; the no-tillage in area of low fertility, in the spring; and, the natural ecosystem, in the summer. As the final mean density of the population of Sminthuroidea, was observed that the no-tillage in area of low fertility went numerically superior to the others, proceeded by the natural ecosystem, no-tillage in area of high fertility, conventional tillage, and, finally, no-tillage in area of medium fertility. Onychiuridae had three populational picks in the no-tillage in area of low fertility: being a larger in the winter and other two, smaller in the summer and in the autumn. In the no-tillage in area of medium fertility presented only a populational pick in the winter. In the other treatments there was not significantly populational picks. In relation to the final mean density of the community of Onychiuridae, the no-tillage plantation in area of low fertility was superior, proceeded by the other treatments of no-tillage, and, in third plan, the natural ecosystem and the conventional tillage.

KEY WORDS. Collembola, Onychiuridae, Sminthuroidea, no-tillage, conventional tillage
\end{abstract}

Atualmente a agricultura tem assumido, de forma crescente, caráter complexo e intensivo, caracterizado por uma exploração do solo que não respeita sua capacidade de uso (FANCELLI \& FAVARIN 1989). Esta prática trouxe sérios prejuízos ao meio ambiente e para o próprio homem. Visando minimizar estes danos, o homem está a procura de um modo de cultivar o solo, que lhe deixe o mais próximo das condições naturais.

1) Curso de Pós-Graduação em Engenharia Florestal, Setor de Ciências Agrárias, Universidade Federal do Paraná. Caixa Postal 2959, 80001-970 Curitiba, Paraná, Brasil.

2) DCA/CEUD, Universidade Federal de Mato Grosso do Sul. Caixa Postal 533, 79804-970 Dourados, Mato Grosso do Sul, Brasil.

3) Departamento de Estatística, Universidade Federal do Paraná. Caixa Postal 19081, 81531-990 Curitiba, Paraná, Brasil. 
O plantio direto, retendo os resíduos vegetais na superficie, imita os ecossistemas naturais: a estrutura do solo permanece, a temperatura e umidade são mais moderadas e assim o seu habitat pode ser mais favorável à fauna do solo (PERDUE \& CROSSLEY 1989). Estas propriedades vinham sendo prejudicadas pelas operações convencionais de preparo de solo (FIAPAR 1981).

Os organismos mais numerosos da mesofauna edáfica são os Oribatei (Acari: Cryptostigmata) e os Collembola, sendo que, juntos, constituem de $72 \%$ a $97 \%$, em número de indivíduos, da fauna total de artrópodos no solo (SINGH \& PILLAI 1975). Segundo DUNGER $(1983,1956)$, a mesofauna edáfica tem um papel de catalizadora da atividade microbiana na decomposição da matéria orgânica, bem como pode ser considerada como decompositora primária e/ou secundária da matéria orgânica.

Dando seqüência à pesquisa iniciada em SAUTTER et al. (1999), o presente trabalho tem como objetivo comparar as comunidades de Sminthuroidea e Onychiuridae (Collembola), entre plantio direto em três níveis de fertilidade, plantio convencional e um ecossistema natural (campo nativo).

\section{MATERIAL E MÉTODOS}

O presente trabalho foi conduzido em diversas propriedades de agricultores, cooperados da Cooperativa Agropecuária Batavo Ltda., região sul do Paraná, segundo planalto paranaense, no município de Carambeí $\left(25^{\circ} 13^{\prime} \mathrm{S}\right.$ e $50^{\circ} 0 \mathrm{l}$ ' $\left.\mathrm{W}\right)$.

Os solos das áreas pesquisadas foram todos caracterizados como Latossolo Vermelho-Escuro (EMBRAPA-SNLCS 1981), cujas características físicas e químicas são apresentadas na tabela I.

Tabela I. Análise química e física dos solos das áreas experimentais: plantio direto em área de baixa (A), média (B) e alta fertilidade (C), plantio convencional (D) e ecossistema natural (campo nativo) (E). Ponta Grossa, Paraná, setembro de 1993.

\begin{tabular}{|c|c|c|c|c|c|c|c|c|c|c|c|}
\hline \multirow{2}{*}{ Tratamentos } & \multirow{2}{*}{$\underset{\mathrm{CaCl}_{2}}{\mathrm{pH}}$} & $\mathrm{Al}^{+3}$ & $\mathrm{H}+\mathrm{Al}$ & $\mathrm{Ca}^{+2}$ & $\mathrm{Mg}^{+2}$ & $\mathrm{~K}^{+}$ & \multirow{2}{*}{$\underset{(\mathrm{ppm})}{\mathrm{P}}$} & \multirow{2}{*}{$\begin{array}{c}\mathrm{C} \\
(\%)\end{array}$} & \multirow{2}{*}{$\begin{array}{c}\text { Areia } \\
(\%)\end{array}$} & \multirow{2}{*}{$\begin{array}{l}\text { Silte } \\
(\%)\end{array}$} & \multirow{2}{*}{$\begin{array}{c}\text { Argila } \\
(\%)\end{array}$} \\
\hline & & \multicolumn{5}{|c|}{ meq $/ 100 \mathrm{~cm}^{3}$ de solo } & & & & & \\
\hline$A$ & 5,6 & 0,0 & 3,6 & 4,4 & 2,7 & 0,46 & 10 & 4,9 & 52 & 14 & 34 \\
\hline B & 6,2 & 0,0 & 2,9 & 7,5 & 3,3 & 0,35 & 27 & 4,4 & 48 & 20 & 32 \\
\hline C & 6,3 & 0,0 & 3,1 & 7,5 & 3,9 & 0,65 & 11 & 4,7 & 28 & 32 & 40 \\
\hline$D$ & 5,4 & 0,0 & 4,6 & 4,2 & 2,5 & 1,05 & 11 & 3,8 & 44 & 14 & 42 \\
\hline$E$ & 4,4 & 0,8 & 7,7 & 1,6 & 1,9 & 0,29 & 2 & 3,8 & 26 & 22 & 52 \\
\hline
\end{tabular}

O clima desta região, conforme FIAPAR (1978) é, segundo a classificação de Koeppen, classificado como Cfb, isto é, subtropical úmido, mesotérmico, verões frescos, geadas severas demasiadamente freqüentes e sem estação seca. A precipitação anual está em torno de 1400 a $1600 \mathrm{~mm}$, umidade relativa de $80 \%$ a $85 \%$ e a temperatura média anual de 17 a $18^{\circ} \mathrm{C}$. A precipitação e a temperatura média mensal durante o período do experimento encontram-se em SAUTTER et al. (1999).

Utilizou-se um delineamento de blocos ao acaso, com cinco tratamentos e três repetições. Em cada tratamento foi marcada, ao acaso, uma área de $45 \times 45 \mathrm{~m}$. Dentro destas áreas, foram marcados, também ao acaso, três blocos (repetições) de $3 \times 3 \mathrm{~m}$, totalizando-se 15 blocos. Estes tratamentos constituíram-se de plantio direto em três níveis de fertilidade, plantio convencional e um ecossistema natural (campo 
nativo). O cronograma de manejo de cada uma das áreas está descrito em SAUTTER et al. (1999).

Os Collembola foram estudados com base em amostragens feitas no período de 21 de outubro de 1992 a 29 de setembro de 1993. Foram realizadas no total 13 amostragens, com período aproximado entre elas de quatro semanas. A cada data de amostragem efetuou-se três amostragens por parcela, perfazendo um total de nove amostras por tratamento e 45 amostras a cada data.

A coleta de amostras e a extração dos Collembola foi feita com o auxílio do método do Funil de Berlese modificado (diâmetro de $4 \mathrm{~cm}$ e profundidade de $5 \mathrm{~cm}$ ), descrito por SAUTTER \& SANTOS (1991). A identificação dos Collembola ao nível de família, foi feita com o auxílio da chave de identificação proposta por PALÁciosVARGAS (1990), para a região Neotropical.

Os dados obtidos da mesofauna, devido à sua heterogeneidade, foram transformados em $\sqrt{x+0,5}$ (GERARD \& BERTHET 1966), sendo depois, submetidos à análise de variância (F-teste), e as médias comparadas pelo teste de Duncan ao nível de $10 \%$ de probabilidade, conforme COCHRAN \& COX (1957).

\section{RESULTADOS E DISCUSSÃO}

\section{Flutuação populacional da comunidade de Sminthuroidea}

A figura 1 mostra que os Sminthuroidea não apresentaram flutuações populacionais grandes, todavia sempre com densidades baixas, nos tratamentos de plantio convencional e plantio direto em área de média fertilidade. $\mathrm{O}$ tratamento de plantio direto em área de baixa fertilidade apresentou um crescimento populacional na primavera de 1993; o plantio direto em área de alta fertilidade, no outono, o que se deve ao fato de que, a partir do inverno até o final do experimento, não ter sido feito nenhum trato cultural nesta área e, o ecossistema natural, no verão, o que deve ser atribuído, provavelmente, às temperaturas mais favoráveis neste período (SAUTTER et al. 1999).

A tabela II mostra a densidade média da população de Sminthuroidea, amostrados nos cinco tratamentos, sendo estas média comparadas pelo teste de Duncan ao nível de $10 \%$ de probabilidade. Em cinco das 13 épocas de coleta constatou-se diferenças estatisticamente significativas. A comunidade de Sminthuroidea foi maior no ecossistema natural em duas épocas de coleta: fevereiro e março. Em maio o plantio direto em área de alta fertilidade, e em final de julho e final de setembro, o plantio direto em área de baixa fertilidade, foram superiores aos demais tratamentos. Conforme a densidade média final da comunidade de Sminthuroidea, observa-se que o plantio direto em área de baixa fertilidade foi numericamente superior aos demais, seguido pelo ecossistema natural, e pelos dois outros tratamentos de plantio direto, e o plantio convencional, o que discorda com os dados de WINTER et al. (1990), que afirmam serem os Sminthuroidea mais numerosos no plantio convencional, que no plantio direto e no ecossistema natural. Este resultado foi encontrado devido ao fato de que esta super-família é formada basicamente de formas epiedáficas (PRICE \& BENHAM 1977), necessitando condições ideais na superfície do solo para sobreviverem, o que o plantio direto proporcionou. 


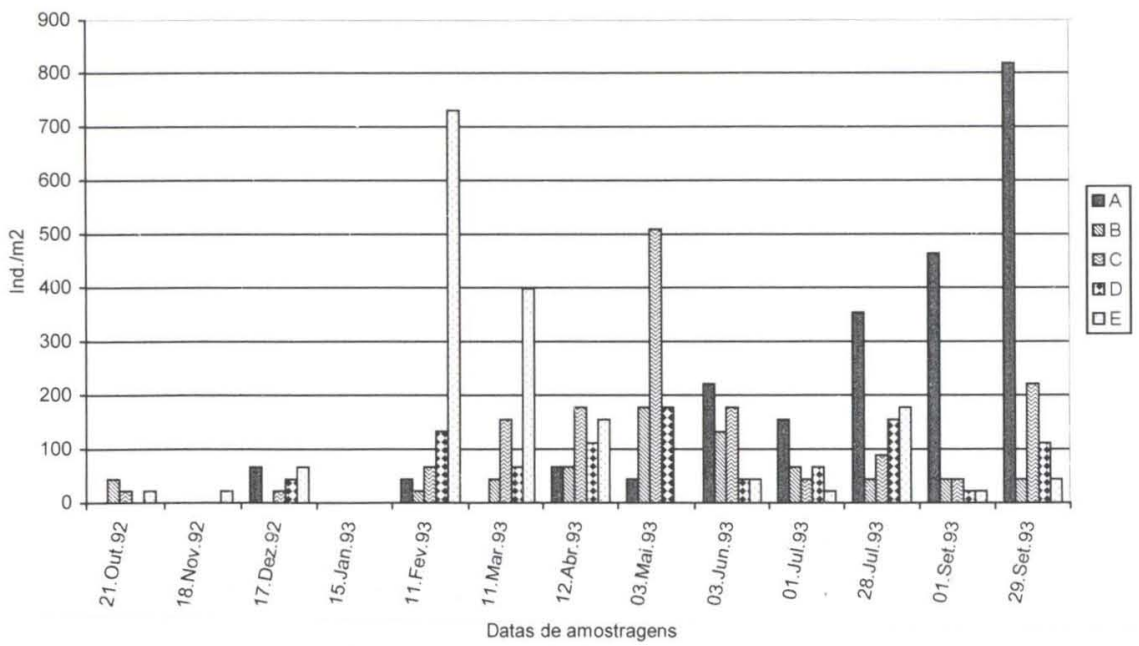

Fig. 1. Flutuação populacional da comunidade de Sminthuroidea em plantio direto em área de baixa (A), média (B) e alta fertilidade (C), plantio convencional (D) e um ecossistema natural (campo nativo) (E). Ponta Grossa, Paraná, 1992/1993.

Tabela II. Sminthuroidea amostrados nos cinco tratamentos: plantio direto em área de baixa (A), média (B) e alta fertilidade (C), plantio convencional (D) e um ecossistema natural (campo nativo) (E). Média de nove amostras, expressa em número de indivíduos por metro quadrado. Ponta Grossa, Paraná, 1992/1993.

\begin{tabular}{|c|c|c|c|c|c|c|}
\hline Datas de amostragens & A & B & C & D & $E$ & Média \\
\hline $21-X-92$ & $\mathrm{Oa}$ & $44 a$ & $22 a$ & $0 a$ & $22 a$ & 17 \\
\hline $18-X 1-92$ & Oa & $\mathrm{Oa}$ & $0 a$ & $\mathrm{Oa}$ & $22 a$ & 4 \\
\hline $17-X \mid 1-92$ & $66 a$ & $\mathrm{Oa}$ & $22 a$ & $44 a$ & $66 a$ & 39 \\
\hline $15-1-93$ & $\mathrm{Oa}$ & $\mathrm{Oa}$ & $\mathrm{Oa}$ & $\mathrm{Oa}$ & $\mathrm{Oa}$ & 0 \\
\hline $11-11-93$ & $44 b$ & $22 b$ & $66 b$ & $133 b$ & $730 a$ & 199 \\
\hline $11-111-93$ & $\mathrm{Ob}$ & $44 b$ & $155 a b$ & $66 b$ & $398 a$ & 132 \\
\hline $12-I V-93$ & $66 a$ & $66 \mathrm{~b}$ & $177 a$ & $111 a$ & $155 a$ & 115 \\
\hline $3-V-93$ & $44 b$ & $177 \mathrm{~b}$ & $509 a$ & $177 b$ & $\mathrm{Ob}$ & 181 \\
\hline $3-\mathrm{VI}-93$ & $221 a$ & $132 a$ & $177 a$ & $44 a$ & $44 a$ & 124 \\
\hline $1-V I I-93$ & $155 a$ & $66 a$ & $44 a$ & $66 a$ & $22 a$ & 70 \\
\hline $28-V I I-93$ & $354 a$ & $44 b$ & $88 b$ & $155 a b$ & $177 a b$ & 164 \\
\hline $1-\mid X-93$ & $464 a$ & $44 a$ & $44 a$ & $22 a$ & $22 a$ & 119 \\
\hline $29-\mid X-93$ & $818 a$ & $44 b$ & $221 b$ & $111 \mathrm{~b}$ & $44 b$ & 248 \\
\hline Média - & 172 & 52 & 117 & 71 & 131 & 109 \\
\hline
\end{tabular}

*) Médias, da mesma linha, seguidas pela mesma letra, não diferem significativamente pelo teste de Duncan, ao nivel de $10 \%$ de probabilidade.

\section{Flutuação populacional da comunidade de Onychiuridae}

Segundo a figura 2, a comunidade de Onychiuridae no tratamento de plantio direto em área de baixa fertilidade apresentou três picos populacionais durante o período do experimento: um, maior, no inverno, e, outros dois, menores, no verão e no outono. A comunidade de Onychiuridae em área de média fertilidade também apresentou um pico populacional no inverno. Os demais tratamentos não apresentaram picos populacionais significativos. 


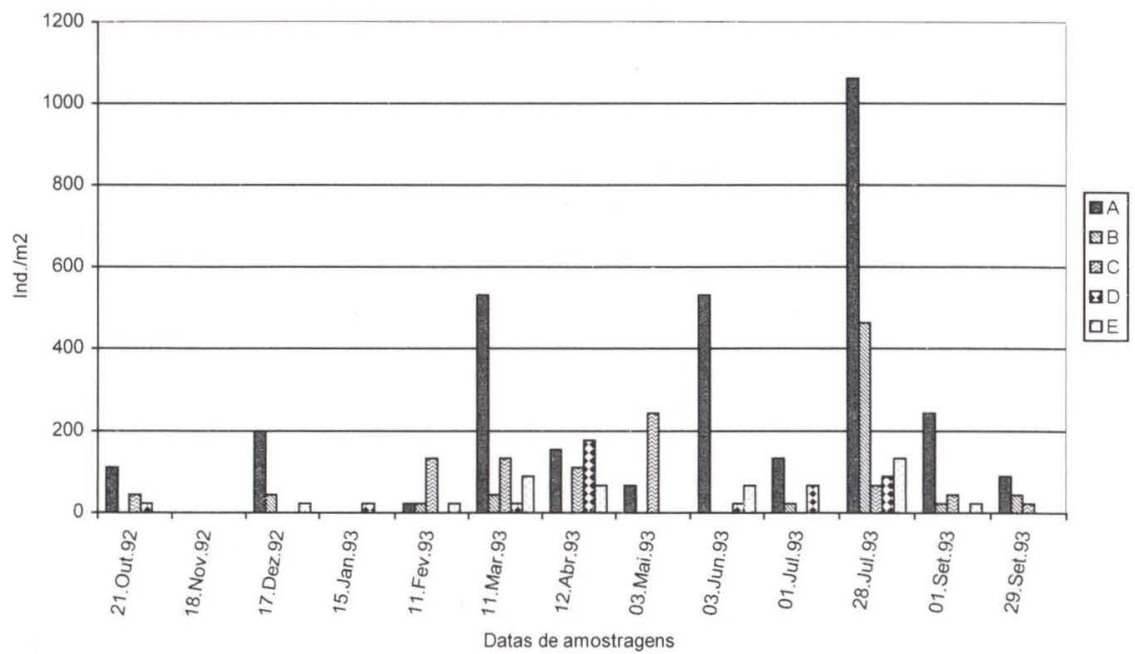

Fig. 2. Flutuação populacional da comunidade de Onychiuridae em plantio direto em área de baixa (A), média (B) e alta fertilidade (C), plantio convencional (D) e um ecossistema natural (campo nativo) (E). Ponta Grossa, Paraná, 1992/1993.

Conforme pode ser visto na tabela III, em quatro das 13 amostragens evidenciou-se diferenças estatisticamente significativas entre os tratamentos, e em todas estas ocasiões o plantio direto foi superior ao plantio convencional e ao ecossistema natural.

Tabela III. Onychiuridae amostrados nos cinco tratamentos: plantio direto em área de baixa (A), média (B) e alta fertilidade (C), plantio convencional (D) e um ecossistema natural (campo nativo) (E). Média de nove amostras, expressa em número de indivíduos por metro quadrado. Ponta Grossa, Paraná, 1992/1993.

\begin{tabular}{|c|c|c|c|c|c|c|}
\hline Datas de amostragens & A & B & C & D & $E$ & Média \\
\hline $21-X-92$ & $111 a$ & $\mathrm{Oa}$ & $44 a$ & $22 a$ & $\mathrm{Oa}$ & 35 \\
\hline $18-X \mid-92$ & $\mathrm{Oa}$ & $\mathrm{Oa}$ & Oa & $\mathrm{Oa}$ & $\mathrm{Oa}$ & 0 \\
\hline $17-X \mid 1-92$ & $199 a$ & $44 a b$ & $\mathrm{Ob}$ & $\mathrm{Ob}$ & $22 b$ & 53 \\
\hline $15-1-93$ & Oa & $\mathrm{Oa}$ & Oa & $22 a$ & $\mathrm{Oa}$ & 4 \\
\hline $11-\mid 1-93$ & $22 b$ & $22 b$ & $133 a$ & $O b$ & $22 b$ & 40 \\
\hline $11-|| 1-93$ & $531 a$ & $44 a$ & $133 a$ & $22 a$ & $88 a$ & 164 \\
\hline $12-I V-93$ & $155 a$ & $0 \mathrm{a}$ & $111 \mathrm{a}$ & $177 \mathrm{a}$ & $66 a$ & 101 \\
\hline $3-V-93$ & $66 a$ & $\mathrm{Oa}$ & $243 a$ & $\mathrm{Oa}$ & $\mathrm{Oa}$ & 62 \\
\hline $3-\mathrm{VI}-93$ & $531 a$ & $\mathrm{Ob}$ & $O b$ & $22 b$ & $66 b$ & 13 \\
\hline $1-\mathrm{VII}-93$ & $133 a$ & $22 a b$ & $O b$ & $66 a b$ & $\mathrm{Ob}$ & 44 \\
\hline $28-V I I-93$ & $1061 a$ & $464 a$ & $66 a$ & $88 a$ & $133 a$ & 362 \\
\hline $1-1 X-93$ & $243 a$ & $22 a$ & $44 a$ & $\mathrm{Oa}$ & $22 a$ & 66 \\
\hline $29-\mid X-93$ & $88 a$ & $44 a$ & $22 a$ & $\mathrm{Oa}$ & $\mathrm{Oa}$ & 31 \\
\hline Média & 201 & 51 & 61 & 30 & 32 & 75 \\
\hline
\end{tabular}

*)Médias, da mesma linha, seguidas pela mesma letra, não diferem significativamente pelo teste de Duncan, ao nível de $10 \%$ de probabilidade.

Na média final dos tratamentos, o resultado indica ser o plantio direto em área de baixa fertilidade superior, seguido pelos outros tratamentos de plantio direto, 
e, em terceiro plano, o ecossistema natural e o plantio convencional. O que concorda com WINTER et al. (1990). A maior densidade dos Onychiuridae no plantio direto em área de baixa fertilidade, pode ser atribuída à maior porcentagem de areia nos solos deste tratamento (Tab. I), dando condições para que esta família, de forma de vida tipicamente euedáfica, se estabelecesse com maior sucesso.

\section{CONCLUSÕES}

Os Sminthuroidea e Onychiuridae foram encontrados em maior número, quando o estágio de desenvolvimento das culturas era adiantado, formando uma boa cobertura do solo.

Os Sminthuroidea e Onychiuridae foram muito sensíveis à falta de chuva em todos os tratamentos, sendo que a maior queda foi no plantio convencional.

A comunidade de Sminthuroidea e Onychiuridae diminuiu muito com a queima da vegetação no ecossistema natural, com restabelecimento relativamente rápido, e este ligado ao restabelecimento da vegetação.

A implantação das culturas, tanto no plantio direto, quanto no convencional, foi uma das principais causas da queda da população.

\section{REFERÊNCIAS BIBLIOGRÁFICAS}

Cochran, W.G. \& G.M. COX. 1957. Experimental designs. New York, John Wiley \& Sons Inc., $2^{\text {nd }}$ ed., 595 p.

DUNGER, W. 1956. Untersuchungen über die Laubstreuzersetzung durch Collembolen. Zool. Jb. Abt. Systematik. 94: 75-98.

- 1983. Tiere im Boden. Die Neue-Brehm Buecherei 327. Wittemberg, A Ziemsen Verlag, 287p.

EMBRAPA-SNLCS. 1981. Levantamento de Reconhecimento dos Solos do Estado

do Paraná. Curitiba, Embrapa/SNLCS, 1 mapa, escala 1:6.000.000..

FANCELLI, A.L.; J.L. FAVARIN. 1989. Realidade e perspectiva para o sistema de plantio direto no Estado de São Paulo, 15-34. In: A.L. FANCELli (Ed.). Plantio Direto no Estado de São Paulo. Piracicaba, Fealq/Esalq/Usp, 190p.

FIAPAR. 1978. Cartas Climáticas do Estado do Paraná. Londrina, Fiapar, 38p. 1981. Plantio Direto no Estado do Paraná. Londrina, Fiapar, 244p.

GERARD, G. \& P.A. BERTHET. 1966. statistical study of microdistribution of Oribatei (Acari). Part II: The transformation of the data. Oikos 17: 142-149.

Palácios-VARGAS, J.G. 1990. Diagnosis y clave para determinar las familias de los Collembola de la Región Neotropical. Manuales y Guias para el Estudo de Microartrópodos. México, Fac. Ciencias (UNAM), 15p.

Perdue, J.C. \& D.A. Crossley JR. 1989. Seasonal abundance of soil mites (Acari) in experimental agroecosystems: Effects of drought in no-tillage and conventional tillage. Soil \& Tillage Res. 15: 117-124.

PRICE, D.W. \& G.S. BENHAM JR. 1977. Vertical distribution of soil inhabiting microarthropods in an agricultural habitat in California. Environ. Entomol. 6 (4): 575-580.

SAUTTER, K.D. \& H.R. DOS SANTOS. 1991. Recuperação de solos degradados pela 
mineração de xisto, tendo como bioindicadores insetos da ordem Collembola. Rev. Set. Ciênc. Agrár. 11 (1-2): 85-91.

SautTer, K.D.; H.R. Dos SANTOS \& P.J. Ribeiro JR. 1999. Comparação das comunidades de Entomobryidae e Isotomidae (Collembola) entre plantio direto em três níveis de fertilidade, plantio convencional e um ecossistema natural (campo nativo) em Ponta Grossa, Paraná, Brasil. Revta. Bras. Zool. 16 (1): 117-124.

Singh, J. \& K.S. PILlaI. 1975. A study of soil microarthropod communities in some fields. Rev. Ecol. Biol. Sol 12 (3): 579-590.

WINTER, J.P.; R.P. VORONEY \& D.A. AINSWORTH. 1990. Soil microarthropods in long term no-tillage and conventional tillage corn production. Can. Jour. Soil. Sci. 70: 641-653.

Recebido em 29.VIII.1997; aceito em 25.I.1999. 